\title{
ON THE ALGEBRAIC CLOSURE OF CERTAIN PARTIALLY ORDERED FIELDS( $\left.{ }^{1}\right)$
}

\author{
BY \\ WALTER STRODT
}

Introduction. This paper is divided into five parts:

Part I. Graduated fields.

Part II. Fields of meromorphic functions.

Part III. Examples for Part II.

Part IV. Applications to algebraic differential equations.

Part V. Appendix.

Part I deals with an abstract algebraic structure called a graduated field, which may be considered as a generalization of a field having a non-Archimedean valuation which is trivial over the prime field. (Cf. Appendix, Note 1.) A graduated field is an ordered quadruple $\left(\mathscr{K}_{0}, \prec, \mathscr{U}, \mathscr{C}_{0}\right)$ in which $\mathscr{K}_{0}$ is a commutative field of characteristic zero, $\prec$ is a partial order relation (cf. Appendix, Note 2) in $\mathscr{K}_{0}, \mathscr{C}_{0}$ is a subfield of $\mathscr{K}_{0}, \mathscr{U}$ is a subgroup of the multiplicative group $\mathscr{K}$ (cf. the notational conventions in Appendix, Note 3), and various hypotheses are made about the interrelations of $\prec$ with $\cdot$ and + in $\mathscr{K}_{0}$, in $\mathscr{C}_{0}$, and in $\mathscr{U}$ $(\$ \$ 1,2)$. In terms of $\prec$ an equivalence relation $\sim$ is introduced into $\mathscr{K}$ by the definition: $f \sim g$ means $f-g \prec g$. Two subgroups $\mathscr{M}$ and $\mathscr{A}$ of $\mathscr{K}$ are defined by setting $\mathscr{M}$ equal to the product group $\mathscr{C} \mathscr{U}$ and by setting $\mathscr{A}=\{f: \exists m \in \mathscr{M}$ such that $f \sim m\}$. Polynomials whose coefficients lie in a field $\mathscr{F}_{0}$ satisfying $\mathscr{M} \subset \mathscr{F}_{0} \subset \mathscr{A}_{0}$ are said to be asymptotically inscribed.

For asymptotically inscribed polynomials $F$ the element $p$ of $\mathscr{M}$ is called a point of stability if $q \sim p$ implies $F(q) \sim F(p)$, a point of instability otherwise. If $p$ is a point of instability for $F$, its multiplicity is defined to be the minimum $s$ such that $p$ is a point of stability for the sth derivative $F^{(s)}$. The principal result in Part I is Theorem I, §8, which states, in effect, that every graduated field having the following two properties (a) and (b):

(a) $\mathscr{C}_{0}$ is algebraically closed, and

(b) whenever an asymptotically inscribed polynomial $F$ has a point of instability $p$, of multiplicity 1 , then $F$ has a root $y$ with $y \sim p$;

must also have the following property (c) as well:

Presented to the Society, June 3, 1961; received by the editors July 5, 1961.

(1) This investigation was supported in part by the Air Office of Scientific Research (Contract No. AF 49(638)-644) and by the National Science Foundation (Contract No. G12984). 
(c) every asymptotically inscribed polynomial of degree $n$ has $n$ roots in $\mathscr{A}_{0}$. (Cf. Appendix, Note 4.)

The crux of the proof of Theorem I is Fundamental Lemma A, §38, which, in the case where $\mathscr{C}_{0}$ is algebraically closed, implies that if $p$ is a point of instability for $F$, of multiplicity $k \geqq 2$, and if, for some $s$ in $\{1,2, \ldots, k-1\}, q$ is a root of $F^{(s)}$ such that $q \sim p$, then the polynomial $F(q+z)$ either has the solution $z=0$ or has a point of instability of multiplicity $\leqq k-1$. Since $F^{(s)}$ is of lower degree than $F$, this lemma makes it possible to obtain ( $\$ 339-50)$ the proof of Theorem I by means of inductive arguments on the degree and the multiplicity.

In Part II, Theorem I is applied to prove a theorem (Theorem II, \$62) on the algebraic closure of certain fields of meromorphic functions. For this application, $\mathscr{K}_{0}$ is the field (cf. Appendix, Note 5) of all functions meromorphic over a system $\bar{N}$ of regions in the complex plane, and $\mathscr{C}_{0}=\mathrm{C}$ (cf. Appendix, Note 6). $\prec$ and $\mathscr{U}$ are obtained by means of constructions (\$§56-58, and §§59-61, 63-64, respectively) which permit a great deal of choice. For the graduated fields created in this way, hypothesis (a) above is automatically satisfied, and an easy use of the implicit function theorem suffices to verify hypothesis (b). The conclusion (c) in this context (in which $\sim$ is always some concept of asymptotic equivalence), then gives, for polynomials whose coefficients have prescribed asymptotic behavior or expansions, very precise information on the asymptotic behavior or expansions of the roots.

In Part III, various explicit choices of $\mathscr{U}$ and $\prec$ are made, to illustrate Theorem II.

A typical choice of $\mathscr{U}(\$ 70)$ causes the multiplicative group $\mathscr{M}$ to be the set of all "logarithmic monomials" $c x^{r_{0}}(\log x)^{r_{1}}(\log \log x)^{r_{2}} \ldots(\log x)^{r_{p}}$ with $c \in \mathbf{C}-\{0\}, r_{j}$ real, and $p$ fixed ( $\mathscr{U}$ then being the set of all such functions with $c=1)$.

In $\S 71$, this choice of $\mathscr{U}$ is fixed, and it is indicated that the aspect of Theorem II changes in a marked, even qualitative, manner, as the choice of $\prec$ is varied.

In Part IV a brief sketch is given of some applications of these theorems and methods to obtain new results in the theory of algebraic differential equations.

\section{Part I. Graduated Fields}

1. Definition. Let $\mathscr{K}_{0}$ be a commutative field of characteristic zero. Let $\prec$ be a partial order of $\mathscr{K}_{0}$ such that

(1.1) If $f \prec g$, then $f \neq g$.

(1.2) $0 \prec 1$.

(1.3) If $f \prec g$ and $h \neq 0$, then $f h \prec g h$.

(1.4) If $f \prec g$ and $h \prec g$ then $f-h \prec g$.

(1.5) If $f<g$ and $h \prec g$ then $f \prec g+h$.

We shall call the ordered pair $\left(\mathscr{K}_{0}, \prec\right)$ a field with asymptotic order. 
2. Definition. Let $\left(\mathscr{K}_{0}, \prec\right)$ be a field with asymptotic order. Let $\mathscr{U}, \mathscr{C}_{0}$ be subsets of $\mathscr{K}_{0}$ such that

(2.1) $\mathscr{U}$ is a subgroup of the multiplicative group $\mathscr{K}$.

(2.2) For every $f \in \mathscr{U}$ and every integer $p \geqq 1$ there is a unique $g \in \mathscr{U}$ such that $g^{p}=f$.

(2.3) $\mathscr{U}$ is completely ordered by $\prec$.

(2.4) $\mathscr{C}_{0}$ is a subfield of $\mathscr{K}_{0}$.

(2.5) If $\{f, g\} \subset \mathscr{K}_{0}$, and $f \prec g$, and $c \in \mathscr{C}_{0}$, then $c f \prec g$.

We shall call the ordered quadruple $\left(\mathscr{K}_{0}, \prec, \mathscr{U}, \mathscr{C}_{0}\right)$ a graduated field.

3. Definition. Let $X=\left(\mathscr{K}_{0}, \prec, \mathscr{U}, \mathscr{C}_{0}\right)$ be a graduated field. Then $f \sim g$ means $f-g \prec g$.

4. Definition. Let $X=\left(\mathscr{K}_{0}, \prec, \mathscr{U}, \mathscr{C}_{0}\right)$ be a graduated field. Then

(4.1) The product group $\mathscr{C} \mathscr{U}$ will be denoted by $\mathscr{M}(X)$ (or, briefly, by $\mathscr{M}$ ).

(4.2) The set $\{f: f \in \mathscr{K}$; there exists $g \in \mathscr{M}$ such that $f \sim g\}$ will be denoted by $\mathscr{A}(x)$ (or, briefly by $\mathscr{A}$ ).

(4.3) Every field $\mathscr{F}_{0}$ such that $\mathscr{M} \subset \mathscr{F}_{0} \subset \mathscr{A}_{0}$ will be called asymptotically constrained over $X$. (Cf. Appendix, Note 7.)

(4.4) If $F(y)=\sum_{i=0}^{n} a_{i} y^{i}$ is a polynomial in $y$, such that $\left\{a_{0}, \ldots, a_{n}\right\}$ is included in a field $\mathscr{F}_{0}$ asymptotically constrained over $X$, then $F$ is said to be asymptotically inscribed over $X$.

5. Definition. Let $F$ be asymptotically inscribed over $X$. Let $p \in \mathscr{M}$. Then $F$ is called stable at $p$ if $F(q) \sim F(p)$ for all $q \in \mathscr{K}_{0}$ such that $q \sim p$; the instability multiplicity of $p$ for $F$ (briefly, inst $(p, F)$ ) is equal to $\min \left\{s: F^{(s)}\right.$ is stable at $\left.p\right\}$; if inst $(p, F) \geqq 1$, then $p$ is called a point of instability for $F$; if $\operatorname{inst}(p, F)=1$, then $p$ is called a simple point of instability for $F$; if inst $(p, F)=0$, then $p$ is called a point of stability for $F$.

6. Definition. The graduated field $X$ is called loosely closed if both the following conditions are satisfied:

(6.1) $\mathscr{C}_{0}$ is algebraically closed.

(6.2) Whenever $F$ is asymptotically inscribed over $X$, and $p$ is a simple point of instability for $F$, there exists a $y_{0} \sim p$ such that $F\left(y_{0}\right)=0$.

7. Definition. The graduated field $X$ is called tightly closed if the following condition holds:

For every field $\mathscr{F}_{0}$ which is asymptotically constrained over $X$ there exists an asymptotically constrained field $\mathscr{G}_{0}$ which is algebraically closed and includes $\mathscr{F}_{0}$.

8. THEOREM I. Let $X$ be a graduated field. Then

(8.1) If $X$ is loosely closed, $X$ is tightly closed.

(8.2) If $X$ is tightly closed, $X$ is loosely closed.

Proof. The proof is given in $\$ 50$. 
9. LemMA. Let $\left(\mathscr{K}_{0}, \prec\right)$ be a field with asymptotic order. Then

(9.1) If $g \neq 0$, then $0 \prec g$.

(9.2) If $f \prec g$, then $g \neq 0$.

(9.3) If $f \prec g$, then $-f \prec g$.

(9.4) If $f \prec g$, and $h \prec g$, then $f+h \prec g$.

(9.5) If $f \prec g$, and $h \prec k$, then $f h \prec g k$.

(9.6) If $f \prec g$, and $f \neq 0$, then $g^{-1} \prec f^{-1}$.

(9.7) If $f \prec g$, then $f \prec f+g$.

Proof. (9.1) follows from (1.2) and (1.3). (9.2) follows from (1.1) if $f=0$, and follows from (9.1), the transitivity of $\prec$, and (1.1) if $f \neq 0$. (9.3) follows from (9.2), (9.1), and (1.4). (9.4) follows from (1.4) and (9.3). (9.5) follows from (9.1), (9.2), and (1.3). (9.6) follows from (1.3), with $h=f^{-1} g^{-1}$. (9.7) follows from (1.5) with $h=f$.

10. Lemma. Let $\left(\mathscr{K}_{0}, \prec\right)$ be a field with asymptotic order. Then

(10.1) If $f \sim g$, then $f \neq 0$ and $g \neq 0$.

(10.2) If $f \neq 0$, then $f \sim f$.

(10.3) If $f \sim g$, then $g \sim f$.

(10.4) If $\sim g$, and $g \sim h$, then $f \sim h$.

(10.5) If $f \sim g$, and $h \sim k$, then $f h \sim g k$.

(10.6) If $\sim g$, then $f^{-1} \sim g^{-1}$.

Proof. (10.1) follows from (9.2), (9.3), and (1.1). (10.2) follows from (9.1). (10.3) follows from (9.7) and (9.3). In (10.4), using (10.3), we have $h \sim g$; hence $f-g \prec g$, and $h-g \prec g$; by (1.4), $f-h \prec g$; by (1.5), $f-h \prec g+(h-g)=h$. In (10.5), $f=g+e$, with $e \prec g$, and $h=k+m$, with $m \prec k$; therefore $f h=g k+t$, with $t=g m+e k+e m$, and $t \prec g k$ by (10.1), (1.3), (9.5), and (9.4). (10.6) follows from (10.1), together with (1.3) applied to $f-g \prec g$ multiplied by $f^{-1} g^{-1}$, together with (10.3).

11. LEMMA. Let $\left(\mathscr{K}_{0}, \prec\right)$ be a field with asymptotic order. Then

(11.1) If $f \prec g$, and $g \sim h$, then $f \prec h$.

(11.2) If $h \sim f$, and $f \prec g$, then $h \prec g$.

(11.3) If $f \prec g$, and $h \sim g$, then $f+h \sim g$.

(11.4) If $f \sim g$, then $f$ is not $\prec g$.

Proof. In (11.1) (using (10.3)), we have $f \prec g$ and $h-g \prec g$; by (1.5), $f \prec g+(h-g)$. In (11.2) we have $f \prec g, h-f \prec f$; by transitivity of $\prec, h-f \prec g$; by (9.4), $f+(h-f) \prec g$. (11.3) follows from (9.4). In (11.4), if we assume the contrary we have $f \prec g$, and, by (10.3), $g \sim f$; then, by (11.1), $f \prec f$, in contradiction to (1.1).

12. GENERAL HYPOTHESIS. In $\S \S 13-50$ the following notations will be fixed: 
$X=\left(\mathscr{K}_{0}, \prec, \mathscr{U}, \mathscr{C}_{0}\right)$ will be a graduated field, and $\mathscr{M}(X), \mathscr{A}(X)$ will be denoted by $\mathscr{M}, \mathscr{A}$, respectively.

13. Lemma. (13.1) $\mathscr{U} \cap \mathscr{C}=\{1\}$.

(13.2) If $g \in \mathscr{M}$, there is a unique ordered pair $(c, u)$ such that $c \in \mathscr{C}, u \in \mathscr{U}$ and $g=c u$.

Proof of (13.1). Obviously $1 \in \mathscr{U} \cap \mathscr{C}$. Suppose $f \in \mathscr{U} \cap \mathscr{C}-\{1\}$. Since $\mathscr{U}$ is completely ordered by $\prec$, either $1 \prec f$ or $f \prec 1$. If $1 \prec f$, then, by (2.5) (since $f \in \mathscr{C}$ ), we have $f \prec f$, in contradiction to (1.1). Suppose $f \prec 1$. Since $f \in \mathscr{U}$, $f^{-1}$ exists, and is in $\mathscr{U}$. Also $f^{-1} \in \mathscr{C}$. By (9.6), $1 \prec f^{-1}$. Hence we are led, by the considerations above, to the contradiction $f^{-1} \prec f^{-1}$.

Proof of (13.2). This is a corollary of (13.1).

14. Lemma. (14.1) If $\{c, d\} \subset \mathscr{C}_{0}$, and $c \prec d$, then $c=0$.

(14.2) If $\{f, g\} \subset \mathscr{K}_{0}$, and $f \prec g$, and $\{c, d\} \subset \mathscr{C}_{0}$, and $d \neq 0$, then $c f \prec d g$.

(14.3) If $\{f, g\} \subset \mathscr{M}$, and $f \sim g$, then $f=g$.

(14.4) For each element $f$ of $\mathscr{A}$ there is a unique element $g$ of $\mathscr{M}$ such that $f \sim g$.

Proof. In (14.1), if $c \neq 0$, then $c^{-1} d$ exists and $\in \mathscr{C}_{0}$; by (2.5) we have $c^{-1} d c \prec d$, contradicting (1.1). In (14.2) we have $c d^{-1} f \prec g$, by (2.5), and then $c f \prec d g$ by (1.3). In (14.3) we have $f=c u, g=d v$, with $\{c, d\} \subset \mathscr{C},\{u, v\} \subset \mathscr{M}$; since $\mathscr{U}$ is completely ordered by $\prec$, either $u \prec v$, or $v \prec u$, or $u=v$; if $u \prec v$, then, by (14.2), $c u \prec d v$, which, by (11.4), contradicts $f \sim g$; similarly $v \prec u$ is impossible, and therefore $u=v$; thus $c u \sim d u$, which, by (10.2) and (10.5), implies $c \sim d$ or $c-d \prec d$; by (14.1), $c-d=0$. In (14.4), the definition of $\mathscr{A}$ implies that there exists a $g \in \mathscr{M}$ such that $f \sim g$; if $h$ is any element of $\mathscr{M}$ such that $f \sim h$, then $g \sim h$ by (10.3) and (10.4); by (14.3), $g=h$.

15. Definition. Let $\{f, g\}<\mathscr{K}_{0}$. We write $f \approx g$ if there exists an element $c$ of $\mathscr{C}$ such that $f \sim c g$, and we write $h \precsim k$ if either $h \prec k$ or $h \approx k$.

16. LEMMA. (16.1) If $f \sim g$, then $f \approx g$.

(16.2) Statements (10.1)-(10.6), (11.1)-(11.4) remain valid if $\sim$ is replaced, everywhere in them, $b y \approx$. (Cf. the notational convention in Appendix, Note 8.)

(16.3) If $\{f, g\} \subset \mathscr{U}$, and $f \approx g$, then $f=g$.

(16.4) If $f \in \mathscr{C}$, then $f \approx 1$.

(16.5) If $f \precsim g$, and $h \precsim g$, then $f+h \precsim g$.

(16.6) If $f \precsim g$, and $g \precsim h$, then $f \precsim h$.

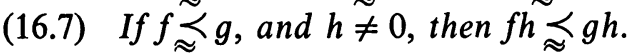

Proof. (16.1) and (16.2) are obvious. In (16.3), $f \sim c g$ for some $c \in \mathscr{C}$; by (14.3), $f=c g$; hence $f g^{-1}=c$; thus $f g^{-1} \in \mathscr{C} \cap \mathscr{U}$; by (13.1), $f g^{-1}=1$. (16.4) is obvious. In (16.5) we have $f=c g+e g, h=d g+s g$ with $\{c, d\} \subset \mathscr{C}_{0}, e \prec 1$, 
$s \prec 1$; hence $f+h=(c+d) g+(s+e) g$, with $c+d \in \mathscr{C}_{0}$ and $s+e \prec 1$ (because of (9.4)); if $c+d=0$, then $f+h \prec g$, while if $c+d \neq 0$, then $f+h \approx g$. (16.6) is a corollary of $(16.2) /[(10.4),(11.1),(11.2)]$ and the transitivity of $\prec$. (16.7) follows at once from (1.3) and (16.2)/[(10.2),(10.5)].

17. Definition. (17.1) Let $f \in \mathscr{A}$. Let $g$ be the unique element of $\mathscr{M}$ such that $f \sim g$ (cf. (14.4)). Let $(c, u)$ be the unique element of $\mathscr{C} \times \mathscr{U}$ such that $g=c u$ (cf. (13.2)). Then $u$ is called the gauge of $f$ and is denoted by $] f[$. We extend this definition by setting: gauge $0=] 0[=0$.

(17.2) Let $f \approx 1$. Then there exists an element $c$ of $\mathscr{C}$ such that $f \sim c$. By (14.4), $c$ is unique. We shall call $c$ the projection of $f$, and denote it by the symbol $\operatorname{proj}(f)$. We extend this definition by writing $\operatorname{proj}(h)=0$ for all $h \prec 1$. (Thus for all $k \precsim 1, \operatorname{proj}(k)$ is the uniquely determined element of $\mathscr{C}_{0}$ such that $k-\operatorname{proj}(k) \prec 1$.)

18. Notation. If $f \in \mathscr{U}$, and $p, q$ are integers with $q \neq 0$, the unique $g \in \mathscr{U}$ such that $g^{q}=f^{p}$ will be denoted by $f^{p / q}$. (Remark: It is easy to see that $f^{p / q}=f^{r / s}$ if $p / q=r / s$.)

19. LEMMA. If $\{f, g\} \subset \mathscr{U}$ and $f \prec g$, then there exists $h \in \mathscr{U}$ such that $f \prec h \prec g$.

Proof. Let $h=(f g)^{1 / 2}$. Then $h^{2}=f g \prec g^{2}$. Hence $h \prec g$. (For $\mathscr{U}$ is completely ordered by $\prec$, and if $h=g$ or $g \prec h$ the inequality $h^{2} \prec g^{2}$ would be impossible.) Similarly $f \prec h$.

20. GENERAL HYPOTHESIS (for $\$ \S 21-37) . \mathscr{F}_{0}$ is a field which is asymptotically constrained over $X, F(y)=\sum_{i=0}^{n} a_{i} y^{i}$ is a polynomial with $\left\{a_{0}, \ldots, a_{n}\right\} \subset \mathscr{F}_{0}$, and $n a_{n} \neq 0$.

21. LEMMA. Assume \$20. Let $p$ be a point of instability for $F$, with inst $(p, F)=s \geqq 1$. Then $F^{\prime}$ is asymptotically inscribed, and $\operatorname{inst}\left(p, F^{\prime}\right)=s-1$.

Proof. Obvious.

22. Lemma. Assume $\$ 20$. Let $h \in \mathscr{F}, w \in \mathscr{M}$. Let $G(z)=h F(w z)$. Then $G$ is asymptotically inscribed, and $\operatorname{inst}(p, F)=\operatorname{inst}(p / w, G)$ for all $p \in \mathscr{M}$.

Proof. $G$ is asymptotically inscribed, since its coefficients lie in $\mathscr{F}_{0}$; the remaining statement is a corollary of the obvious relation $G^{(j)}(z)=h w^{j} F^{(j)}(w z)$.

23. Lemma. Assume §20. Let $p \in \mathscr{M}$. Let $m=\max \{] a_{i} p^{i}[: i \geqq 0\}$. (We remark that because of (10.5), $a_{i} p^{i} \in \mathscr{A}_{0}$.) Then

(23.1) If $q \sim p$, and $j$ is any non-negative integer, $q^{j} F^{(j)}(q) \precsim m$.

(23.2) If $q \sim p$, then inst $(p, F)=\min \left\{j: q^{j} F^{(j)}(q) \approx m\right\}$.

Proof of (23.1). This follows at once from (10.5), (16.1), (16.6), and (16.5).

Proof of (23.2). Let $c_{i}=a_{i} p^{i} m^{-1}$. Then $c_{i} \precsim 1$, and $c_{i} \in \mathscr{F}_{0}$.

Let $\sigma \prec 1$. Let $q=p(1+\sigma)$. Then $q \sim p$. Also, for every non-negative integer $j$ we have $q^{j} F^{(j)}(q)=m(1+\sigma)^{j}$. $G^{(j)}(1+\sigma)$, where $G(z)=\sum_{i=0}^{n} c_{i} z^{i}$. 
Let $\gamma_{i}=\operatorname{proj}\left(c_{i}\right)$, and let $\varepsilon_{i}=c_{i}-\gamma_{i}$. Then $G=H+K$, where $H(z)=\sum_{i=0}^{n} \gamma_{i} z^{i}$ and $K(z)=\sum_{i=0}^{n} \varepsilon_{i} z^{i}$. Let $s=\min \left\{r: H^{(r)}(1) \neq 0\right\}$. (Then $0 \leqq s \leqq s_{0}$, where $s_{0}$ $=\max \{i:] a_{i} p^{i}[=m\}$.) Evidently if $j \leqq s$, then $H^{(j)}(1+\sigma) \sim[(s-j) !]^{-1}$ $\sigma^{s-j} H^{(s)}(1)$. In particular, $H^{(s)}(1+\sigma) \sim H^{(s)}(1)$. Now $\varepsilon_{i} \in \mathscr{F}_{0}$ and $\varepsilon_{i} \prec 1$. Let $\delta$ $=\min \{] \varepsilon_{i}[: i \geqq 0\}$. Then $\delta \prec 1$, and $\delta \in \mathscr{U}_{0}$. If $\delta=0$, then $K^{(j)}(1+\sigma)=0$ for all $\sigma$. If $\delta \neq 0$, then $K^{(j)}(1+\sigma) \precsim \delta$ for all $\sigma \prec 1$. in either case, $K^{(j)}(1+\sigma)$ $\prec 1$ for all $\sigma \prec 1$.

It follows that $G^{(s)}(1+\sigma) \sim H^{(s)}(1)$ for all $\sigma \prec 1$, and therefore that $q^{s} F^{(s)}(q)$ $\sim m H^{(s)}(1)$ for all $q \sim p$. This establishes the stability of $F^{(s)}$ at $p$, and the relation $q^{s} F^{(s)}(q) \approx m$. Also, if $j \leqq s-1$, then $H^{(j)}(1+\sigma) \prec 1$, so $G^{(j)}(1+\sigma) \prec 1$, and therefore $q^{j} F^{(j)}(q) \prec m$. Thus $s=\min \left\{j: q^{j} F^{(j)}(q) \approx m\right\}$.

If $\delta=0$, then $K=0$, so $p^{j} F^{(j)}(p)=m H^{(j)}(1)=0$ for $j \leqq s-1$, and therefore $p$ is a point of instability for $F^{(j)}$, if $j \leqq s-1$. If $\delta \neq 0$, then $\delta \in \mathscr{U}$, and therefore we may (and do) take $\sigma \in \mathscr{U}$ such that $\delta^{1 / n} \prec \sigma \prec 1$ (cf. §19). Then, if $j \leqq s-1$, we have $H^{(j)}(1+\sigma) \sim \lambda_{j}$, where $\lambda_{j}=[(s-j) !]^{-1} \sigma^{s-j} H^{(s)}(1)$. But $K^{(j)}(1+\sigma)$ $\prec \sigma^{n} \precsim \sigma^{s-j}$. Hence $G^{(j)}(1+\sigma) \sim \lambda_{j}$, and therefore $q^{j} F^{(j)}(q) \sim m \lambda_{j}$. If $q_{0}$ $=p(1+2 \sigma)$, then by the same argument $\left(q_{0}\right)^{j} F^{(j)}\left(q_{0}\right) \sim m \lambda_{2} 2^{s-j}$, which is not $\sim q^{j} F^{(j)}(q)$, although $q_{0} \sim p$ and $q \sim p$. Thus $F^{(j)}$ is not stable at $p$, if $j \leqq s-1$. Therefore $s=\operatorname{inst}(p, F)$, and from above we have $s=\min \left\{j: q^{j} F^{(j)}(q) \approx m\right\}$. This establishes (23.2).

24. Lemma. Assume $\$ 20$. Let $q \sim p \in \mathscr{M}$. Let $G(y)=(y-q) F(y)=\sum_{i=0}^{n+1} b_{i} y^{i}$. Let $G$ be asymptotically inscribed. Then

(24.1) $\operatorname{inst}(p, G)=1+$ inst $(p, F)$.

(24.2) If $r \in \mathscr{M}-\{p\}$, then inst $(r, G)=\operatorname{inst}(r, F)$.

Proof of (24.1). We have $b_{i}=a_{i-1}-q a_{i}$. Hence $b_{i} p^{i}=a_{i-1} p^{i}-a_{i} q p^{i}$. Let $m=\max \{] a_{i} p^{i}[: i \geqq 0\}, m^{*}=\max \{] b_{i} p^{i}[: i \geqq 0\}$. Let $k=\min \{i:] a_{i} p^{i}[=m\}$. Then $a_{k-1} p^{k} \prec m p$, and $a_{k} q p^{k} \approx m p$. Hence $b_{k} p^{k} \approx m p$. Also $b_{i} p^{i} \lesssim m p$ for all $i$. Thus $m^{*}=m(] p[)$.

Let $\phi_{j}=p^{j} F^{(j)}(p), \gamma_{j}=p^{j} G^{(j)}(p)$. Then $\gamma_{j}=(p-q) \phi_{j}+j p \phi_{j-1}$. Let $t=\operatorname{inst}(p, F)$. Then $\phi_{j} \prec m$ for $j \leqq t-1, \phi_{t} \approx m, \phi_{t+1} \precsim m$. Also $p-q \prec p$. Hence $\gamma_{j} \prec p m$ $\approx m^{*}$ if $j \leqq t$, and $\gamma_{t+1} \approx p m \approx m^{*}$. Therefore, by $\S 23$, inst $(p, G)=t+1$. This proves (24.1).

Proof of (24.2). This is similar to the proof of (24.1).

25. Lemma. Assume \$20. Let $k$ be an integer $\geqq 1$. Let $G(y)=y^{k} F(y)$ Then for every $p \in \mathscr{M}$, we have inst $(p, G)=\operatorname{inst}(p, F)$.

Proof. It suffices to consider the case $k=1$. The argument for that special case is similar to that for (24.1).

26. Lemma. Assume $\$ 20$. Let $p \in \mathscr{M}$. Let $y_{1} \sim p, y_{2} \sim p, y_{1} \neq y_{2}$. Then (26.1) If $F\left(y_{1}\right)=0$, inst $(p, F) \geqq 1$. 
(26.2) If $F\left(y_{1}\right)=F\left(y_{2}\right)=0$, inst $(p, F) \geqq 2$.

Proof of (26.1). Assume the contrary. Then $p$ is a point of stability for $F$. Hence $F\left(y_{1}\right) \sim F(p)$, and therefore, by (10.1), we have the contradiction $F\left(y_{1}\right) \neq 0$.

Proof of (26.2). Assume the contrary. Then, using (26.1), we have inst $(p, F)=1$. Let $G(z)=F\left(y_{1}+z\right)=\sum b_{i} z^{i}$. Let $m=\max \{] a_{i} p^{i}[: i \geqq 0\}$. We have $b_{0}=F\left(y_{1}\right)$ $=0$. By $\$ 23, b_{1} \approx m p^{-1}$, and $b_{j} \precsim m p^{-j}$ for $j \geqq 2$. Let $z_{1}=y_{2}-y_{1}$. Then $z_{1} \neq 0$, and $G\left(z_{1}\right)=0$. Hence $\left(^{*}\right) \stackrel{-}{-} b_{1}=\sum_{i=2}^{n} b_{i} z_{1}^{i-1}$. But $-b_{1} \approx m p^{-1}$, while since $z_{1} \prec p$ we have $b_{i} z_{1}^{i-1} \prec m p^{-i} p^{i-1}=m p^{-1}$ for $i \geqq 2$. Thus we have the contradiction that the two members of $(*)$ cannot be equal.

27. Definition. Assume $\S 20$. Assume $a_{0} \neq 0$. Let $u \in \mathscr{U}$. Let $(r, s)$ be an ordered pair of integers such that $0 \leqq r<s \leqq n$. Then the ordered triple $(r, s, u)$ is called $a$ Newton triple for $F$, over $X$ (or, briefly, a Newton triple for $F$ ), if

(27.1) $a_{i} u^{i} \precsim a_{r} u^{r} \approx a_{s} u^{s}$

for all $i$ such that $0 \leqq i \leqq n$, with the following strict inequality

(27.2) $a_{i} u^{i} \prec a_{r} u^{r}$

holding for all $i \leqq r-1$ and for all $i \geqq s+1$.

28. Lemma. Assume §20. Assume $a_{0} \neq 0$. Then there exists a unique finite sequence $\left(\tau_{1}, \tau_{2}, \ldots, \tau_{k}\right)$ of Newton triples for $F$, with $\tau_{j}=\left(r_{j}, s_{j}, u_{j}\right)$, such that $u_{1} \prec u_{2} \prec \ldots \prec u_{k}$, and such that $\sum_{j=1}^{k}\left(s_{j}-r_{j}\right)=n$. For every Newton triple $\sigma$ there exists a unique $j$ such that $\sigma=\tau_{j}$. The sequence $\left(\tau_{1}, \ldots, \tau_{k}\right)$ can be constructed as follows: Let $\left.b_{i}=\right] a_{i}\left[,(i=0,1, \ldots, n)\right.$. For $b_{h} \neq 0, i \neq h$, let $c_{h i}=\left(b_{i} / b_{h}\right)^{1 /(h-i)}$. Let $r_{1}=0, u_{1}=\min \left\{c_{0 i}: i \geqq 1\right\}, s_{1}=\max \left\{i: c_{0 i}=u_{1}\right\}$. When $\left(\tau_{1}, \ldots, \tau_{q}\right)$ has been determined: if $s_{q} \leqq n-1$ let $r_{q+1}=s_{q}$, let $u_{q+1}=$ $\min \left\{c_{\sigma i}: i>\sigma=r_{q+1}\right\}$ and let $s_{q+1}=\max \left\{i: c_{\sigma i}=u_{q+1}\right\}$; of $s_{q}=n$, let $k=q$.

Proof. The argument follows closely the classic discussion of Newton polygons. We remark, for future application, that in the construction above (in which $u_{j}$ is asserted to increase with $\left.j\right), r_{j}$ obviously increases with $j$.

29. Notation. The sequence $\left(\tau_{1}, \tau_{2}, \ldots, \tau_{k}\right)$ described in $\$ 28$ will be called the Newton sequence for $F$, over $X$.

30. Lemma. Assume §20. Let $a_{o} \neq 0$. Let $\phi \in \mathscr{F}, w \in \mathscr{U}$. Let $G(z)=\phi F(w z)$ $=\sum_{i=0}^{n} b_{i} z^{i}$. Then $G$ is asymptotically inscribed, and if $\left(\tau_{1}, \ldots, \tau_{k}\right)$ is the Newton sequence for $F$, with $\tau_{j}=\left(r_{j}, s_{j}, u_{j}\right)$, then the Newton sequence for $G$ is $\left(\sigma_{1}, \ldots, \sigma_{k}\right)$, with $\sigma_{j}=\left(r_{j}, s_{j}, w^{-1} u_{j}\right)$.

Proof. Since the coefficients of $G$ belong to $\mathscr{F}_{0}, G$ is asymptotically inscribed. Also $b_{0} b_{n}=a_{0} a_{n} w^{n} \phi^{2} \neq 0$. Hence $G$ has a Newton sequence. The remaining conclusions follow from the definition of Newton triple.

31. Lemma. Assume $\$ 20$. Let $a_{0} \neq 0$. Let $(r, s, u)$ be a Newton triple for $F$. Let $G(z)=F(u z)\left(a_{r} u^{r}\right)^{-1}=\sum_{i=0}^{n} c_{i} z^{i}$. Then

(31.1) $1=c_{r}$. 
(31.2) $c_{i} \precsim c_{r}(i=0,1, \ldots, n)$, with strict inequality if $i \leqq r-1$ and if $i \geqq s+1$. (31.3) $c_{r} \approx c_{s}$.

Proof. Obviously, $c_{r}=1$. By $\S 30, G$ has the Newton triple $(r, s, 1)$. That is, (31.2) and (31.3) are valid.

32. Notation. Under the hypotheses of $\S 31$, let $\gamma_{i}=\operatorname{proj}\left(c_{i}\right)$. Then the polynomial $H(z)=\sum_{i=0}^{n} \gamma_{i} z^{i}$ is called the Newton polynomial for $F$ associated with $(r, s, u)$, and the ordered quadruple $(r, s, u, H)$ is called a Newton quadruple for $F$.

The remaining definitions in this section are given under the assumption that $\mathscr{C}_{0}$ is algebraically closed.

For each nonzero root $\zeta$ of $H$ (evidently $\zeta \in \mathscr{C}$ ), the element $m=\zeta u$ of $\mathscr{M}$ is called an approximate zero of $F$, and the multiplicity of $\zeta$ as a root of $H$ is called the approximate-zero multiplicity of $m$ for $F$, and is denoted by $\operatorname{azm}(m, F)$.

If $\operatorname{azm}(m, F)=1$, then $m$ is called a simple approximate zero of $F$.

The assertion $\operatorname{azm}(m, F)=0$ is understood to mean that $m \in \mathscr{M}$ and that $m$ is not an approximate zero of $F$.

33. Lemma. Assume §20. Assume $a_{0} \neq 0$. Assume $\mathscr{C}_{0}$ is algebraically closed. Let $\left\{w_{1}, w_{2}, \ldots, w_{a}\right\}$ be the set of all approximate zeros of $F$. Let $k_{j}=\operatorname{azm}\left(w_{j}, F\right)$. Then $\sum_{j=1}^{a} k_{j}=n$.

Proof. Let $(r, s, u, H)$ be a Newton quadruple. Let $\left\{\zeta_{1}, \ldots, \zeta_{b}\right\}$ be the set of all nonzero roots of $H$. Let $h_{i}$ be the multiplicity of $\zeta_{i}$ as a root of $H$. Then, by $\S 31$, $\sum h_{i}=s-r$. Let $v_{i}=\zeta_{i} u$. Then $\operatorname{azm}\left(v_{i}, F\right)=h_{i}$. Hence $\sum \operatorname{azm}\left(v_{i}, F\right)=s-r$. That is, $\Sigma\left\{\operatorname{azm}\left(w_{j}, F\right):\right] w_{j}[=u\}=s-r$. Hence

$$
\sum_{j=1}^{s} \operatorname{azm}\left(w_{j}, F\right)=\sum\{s-r: \text { all Newton triples }(r, s, u)\}=n
$$

(by $\$ 28)$.

34. Notation. Assume $\S 20$. Assume $a_{0} \neq 0$. Assume $\mathscr{C}_{0}$ is algebraically closed. Let $S=\left(p_{1}, p_{2}, \ldots, p_{n}\right)$ be an ordered $n$-tuple of elements of $\mathscr{M}$. We shall call $S$ a sequence of approximate solutions, multiplicities counted, for $F$, if for every $q \in \mathscr{M}$ the number of integers $i$ in the set $\{1,2, \ldots, n\}$ such that $p_{i}=q$ is equal to $\operatorname{azm}(q, F)$.

35. Lemma. Assume §20. Assume $a_{0} \neq 0$. Assume $\mathscr{C}_{0}$ is algebraically closed. Let $(r, s, u, H)$ be a Newton quadruple for $F$. Let $\phi \in \mathscr{F} ; w \in \mathscr{U}$. Let $G(z)=\phi F(w z)$. Then $(r, s, u / w, H)$ is a Newton quadruple for $G$, and for every $\zeta \in \mathscr{C}$ we have $\operatorname{azm}(\zeta u / w, G)=\operatorname{azm}(\zeta u, F)$.

Proof. This follows immediately from $\S 30$ and the definitions of Newton quadruple and azm.

36. Lemma. Assume $\S 20$. Assume $a_{0} \neq 0$. Assume $\mathscr{C}_{0}$ is algebraically closed. Let $p \in \mathscr{M}$. Then inst $(p, F)=\operatorname{azm}(p, F)$. 
Proof. Let $p \in \mathscr{M}$. Let $\left.q_{i}=\right] a_{i} p^{i}\left[\right.$. Let $m=\max \left\{q_{i}: i \geqq 0\right\}$. Let $J=\left\{i: q_{i}\right.$ $=m\}$. Let $r=\min \{i: i \in J\}, s=\max \{i: i \in J\}$.

CASE I: $r=s$. Then $J$ has only one element, $r$. Also, $a_{r} p^{r} \approx m$, while $a_{i} p^{i} \prec m$ for all $i \neq r$. Hence $F(p) \approx m$, and therefore, by $\S 23$, inst $(p, F)=0$. Suppose $\operatorname{azm}(p, F) \geqq 1$. Let $u=] p[$. Then there exists a Newton triple $(\rho, \sigma, u)$ for $F$. It follows that $a_{r} u^{r} \precsim a_{\rho} u^{\rho} \approx a_{\sigma} u^{\sigma}$, and therefore that $m \precsim q_{r} \precsim q_{\rho} \approx q_{\sigma} \approx m$, which implies that $\{\rho, \sigma\} \subset J$, although $J$ has only one element. This contradiction shows that $\operatorname{azm}(p, F)=0$. Thus, in Case $\mathrm{I}$, inst $(p, F)=\operatorname{azm}(p, F)$.

CASE II: $r \leqq s-1$. Let $u=] p\left[, \zeta=p / u\right.$. Then $a_{r} u^{r} \approx a_{s} u^{s}$, and $a_{i} u^{i} \precsim a_{r} u^{r}$ for all $i$, with strict inequality if $i \leqq r-1$ and if $i \geqq s+1$. Thus $(r, s, u)$ is a Newton triple for $F$. Let $(r, s, u, H)$ be a Newton quadruple for $F$. Let $G(z)=$ $F(u z)\left(a_{r} u^{\prime}\right)^{-1}=\sum_{i=0}^{n} c_{i} z^{i}$, let $\gamma_{i}=\operatorname{proj}\left(c_{i}\right), \varepsilon_{i}=c_{i}-\gamma_{i}, E(z)=\sum_{i=0}^{n} \varepsilon_{i} z_{i}$. Then $G=H+E$. Let $\operatorname{azm}(p, F)=k \geqq 0$. Then $H^{(j)}(\zeta)=0$ if $j \leqq k-1$ and $H^{(k)}(\zeta)$ $\neq 0$. But $E^{(j)}(\zeta) \prec 1$ for all $j$, since $\varepsilon_{i} \prec 1$. Hence $G^{(j)}(\zeta) \prec 1$ if $j \leqq k-1$, while $G^{(k)}(\zeta) \approx 1$. But, by $\S 31,1=\max \{] c_{i} \zeta^{i}[: i \geqq 0\}$. Hence, by $\S 23$, inst $(\zeta, G)$ $=k$. But then, by $\S 22$, inst $(p, F)=k$. Therefore, in Case II, inst $(p, F)=\operatorname{azm}(p, F)$.

37. Lemma. Assume $§ 20$. Assume $\mathscr{C}_{0}$ is algebraically closed. Let $\left\{p_{1}, \ldots, p_{s}\right\}$ be the set of all points of instability for $F$, and let $k_{i}=\operatorname{inst}\left(p_{i}, F\right)$. Let $\delta=\min \left\{j: F^{(j)}(0) \neq 0\right\}$. Then $\delta+\sum_{i=1}^{s} k_{i}=n$.

Proof. This is a corollary of $\S \S 25,33$, and 36 .

38. Fundamental Lemma A. Let $F(y)=\sum_{i=0}^{n} a_{i} y^{i}$ be asymptotically inscribed, with $a_{n} \neq 0$. Let $p$ be a point of instability for $F$, with instability multiplicity $k \geqq 1$. Let $q$ be such that $q \sim p$, and such that the polynomial $G(z)$ $=F(q+z)$ is asymptotically inscribed. Then

(38.1) For every element $v$ of $\mathscr{M}$ such that $v \prec p$, the instability multiplicity $h$ of $v$ for $G$ satisfies $h \leqq k$, with $h \leqq k-1$ holding in the special case where for some $\sigma$ in $\{0,1, \ldots, k-1\}$ we have $F^{(\sigma)}(q)=0$.

(38.2) If $\mathscr{C}_{0}$ is algebraically closed, and $w_{1}, w_{2}, \ldots, w_{T}$ are the points of instability of $G$ such that $w_{i} \prec p$, and if $\lambda_{i}=\operatorname{inst}\left(w_{i}, G\right)$, and $\delta=\min \left\{i: G^{(i)}(0) \neq 0\right\}$, then $\delta+\sum_{i=1}^{T} \lambda_{i}=k$.

Proof of (38.1) Let $G(z)=\Sigma b_{j} z^{j}$. Let $m=\max \{] a_{i} p^{i}[: i \geqq 0\}$. We have $b_{j}=(j !)^{-1} F^{(j)}(q)$. Hence, by $\S 23$, we have $p^{j} b_{j} \precsim m$, with $\prec$ for $j \leqq k-1$, and with $\approx$ for $j=k$. Let $m^{*}=\max \{] b_{j} v^{j}[: j \geqq 0\}$. Let $c_{j}=b_{j} v^{j} / m^{*}$. Then $c_{j} \precsim 1$. Let $\gamma_{j}=\operatorname{proj}\left(c_{j}\right)$.

Now $b_{k} v^{k} \approx m(v / p)^{k}$, so $m(v / p)^{k} \precsim m^{*}$. If $j \geqq k+1$, then $b_{j} v^{j} \precsim m(v / p)^{j}$ $\prec m(v / p)^{k} \precsim m^{*}$, so $c_{j} \prec 1$ if $j \geqq k+1$.

Let $d_{i} \stackrel{\approx}{=} \sum_{j=i}^{n} j(j-1) \ldots(j-i+1) c_{j}$. By $\quad(23.2), v^{h} G^{(h)}(v) \approx m^{*}$. That is, $\sum_{j=h}^{n} j(j-1) \ldots(j-h+1) b_{j} v^{j} \approx m^{*}$, whence $d_{h} \approx 1$. Since $c_{j} \prec 1$ for $j \geqq k+1$, it follows at once that $h \leqq k$. 
Suppose $h=k$. Then $d_{k} \approx 1$. Also, by $(23.2), v^{i} G^{(i)}(v) \prec m^{*}$ if $i \leqq k-1$. Therefore $d_{i} \prec 1$, for $i \leqq k-1$.

Let $\tau=\operatorname{proj}\left(d_{k}\right)$. Let $\lambda_{j}=\gamma_{j} / \tau$. Then $\lambda_{j}=0$ if $j \geqq k+1$. Hence we have $\sum_{j=i}^{k} j(j-1) \ldots(j-i+1) \lambda_{j}=\delta_{i k}(j=0,1, \ldots, k)$. This is a linear system in $\lambda_{0}, \ldots, \lambda_{k}$ with nonvanishing determinant $0 ! 1 ! \ldots k$ !. Hence its solution is unique. But obviously the following is a solution $\left({ }^{* *}\right) \lambda_{j}=(-1)^{k-j}[j !(k-j) !]^{-1}$ (as we see by defining $\phi(x)=(k !)^{-1}(x-1)^{k}$ and expanding the equations $\left.\phi^{(i)}(1)=\delta_{i k}\right)$.

If now for some $\sigma$ in $\{0,1, \ldots, k-1\}$ we have $F^{(\sigma)}(q)=0$, then $0=b_{\sigma}=c_{\sigma}$ $=\gamma_{\sigma}=\lambda_{\sigma}$. But the relation $\lambda_{\sigma}=0$ contradicts $\left({ }^{* *}\right)$. This contradiction shows that in this special case we must have $h \leqq k-1$.

Proof of (38.2). Let $G(z)=z^{\delta} J(z)$. Then $J(0) \neq 0$. Obviously $\delta \leqq k$, since $b_{k} \neq 0$. If $\delta=n$, then $\delta=k=n$, and (38.2) is a trivial consequence of $\$ 25$. Assume $\delta \leqq n-1$. Let $J(z)=\Sigma h_{i} z^{i}$. Then $h_{i}=b_{i+\delta}$. Let $(r, s, u)$ be a Newton triple for $J$ such that $r \leqq k-\delta<s$. Then $h_{k-\delta} u^{k-\delta} \precsim h_{r} u^{r} \approx h_{s} u^{s}$, or $b_{k} u^{k-\delta} \precsim b_{r+\delta} u^{r}$ $\approx b_{s+\delta} u^{s}$. Now $b_{k} \approx m p^{-k}$ and $b_{s+\delta} \precsim m p^{-s-\delta}$. Hence $m p^{-k} u^{k-\delta} \precsim m p^{-s-\delta} u^{s}$, and therefore $(u / p)^{k-\delta-s} \precsim 1$. Since $k-\delta-s \leqq-1$, this implies that $p \precsim u$. Now $b_{r+\delta} \precsim m p^{-r-\delta}$ with strict inequality if $r+\delta \leqq k-1$. Hence $m p^{-k} u^{k-\delta} \precsim m p^{-r-\delta} u^{r}$ with strict inequality if $r+\delta \leqq k-1$. Therefore $(u / p)^{k-\delta-r} \precsim 1$, with strict inequality if $r+\delta \leqq k-1$. But since $p / u \precsim 1$, this means that $r+\delta=k$.

Thus there is a Newton triple $(k-\delta, s, u)$ for $J$, and $p \precsim u$.

Now if $t$ is such that $(t, k-\delta, v)$ is a Newton triple for $J$, we have $h_{t} v^{t} \approx h_{k-\delta} v^{k-\delta}$, or $b_{t+\delta} v^{t} \approx b_{k} v^{k-\delta}$, and since $b_{k} \approx m p^{-k}$ while $b_{t+\delta} \prec m p^{-t-\delta}$, we have $m p^{-k} v^{k-\delta} \prec m p^{-t-\delta} v^{t}$, or $(v / p)^{k-\delta-t} \prec 1$, whence $v \prec p$. Hence all Newton triples for $J$ of the form $(\alpha, \beta, w)$ with $\beta \leqq k-\delta$ have $w \prec p$. It follows that if $\left(r_{1}, r_{2}, \ldots, r_{n-\delta}\right)$ is a sequence of approximate solutions, multiplicities counted, for $J$, and $r_{1} \precsim r_{2} \precsim \ldots \precsim r_{n-\delta}$ we have $r_{k-\delta} \prec p$ while $p \precsim r_{k-\delta+1}$. Hence (by $\S \S 36$ and 25) we have $\sum_{i=1}^{T} \lambda_{i}=k-\delta$. This establishes (38.2).

39. Definition. Let $F$ be asymptotically inscribed and of degree $\geqq 1$. We shall say that $F$ is normal if every asymptotically constrained field $\mathscr{F}_{0}$ containing the coefficients of $F$ has an asymptotically constrained extension containing at least one root of $F$.

If $F$ is asymptotically inscribed and of degree $\geqq 1$, we shall say that $F$ is $a b$ normal if $F$ is not normal.

40. LEMMA. If $F$ is asymptotically inscribed and $F$ is of the first degree, then $F$ is normal.

Proof. Obvious.

41. Definition. Let $X$ be a graduated field. Let $n$ be an integer $\geqq 1$. We shall say that $n$ is regular for $X$ if every polynomial asymptotically inscribed over $X$ whose degree is $\geqq 1$ but $\leqq n-1$ is normal.

42. Lemma. Let $n$ be regular for $X$. Let $G$ be asymptotically inscribed, 
of degree $n$, and normal. Let $\mathscr{F}_{0}$ be any asymptotically constrained field containing the coefficients of $G$. Then there exists an extension $\mathscr{G}_{0}$ of $\mathscr{F}_{0}$ such that $G(y)=c\left(y-y_{1}\right) \ldots\left(y-y_{n}\right)$, with $\left\{y_{1}, \ldots, y_{n}\right\} \subset \mathscr{G}_{0}$.

Proof. Since $G$ is normal, there exists an asymptotically constrained extension $\mathscr{G}_{10}$ of $\mathscr{F}_{0}$ such that $G$ has a root $y_{1}$ in $\mathscr{G}_{10}$. Therefore $G(y)=\left(y-y_{1}\right) H(y)$, where $H$ has coefficients in $\mathscr{G}_{10}$. Since $H$ is asymptotically inscribed, and (degree $H$ ) $=n-1, H$ is normal, and the degree of $H$ is regular for $X$, unless (degree $H$ ) $=0$. If (degree $H)=0$, we have the required factorization with $c=H(y)$. If (degree $H$ ) $\geqq 1, H$ may be treated in the same way as $G$. Repetition of this procedure produces the required factorization.

43. Lemma. Let $n$ be regular for $X$. Let $F$ be abnormal and of degree $n$. Let $s \in\{1,2, \ldots, n-1\}$. Let $y_{1}$ be an element of $\mathscr{K}_{0}$ such that $F^{(s)}\left(y_{1}\right)=0$, and let $G(z)=F\left(y_{1}+z\right)$. Then $G$ is abnormal.

Proof. Assume the contrary. Let $\mathscr{F}_{0}$ be any asymptotically constrained field containing the coefficients of $F$. Then $\mathscr{F}_{0}$ contains the coefficients of $F^{(s)}$. Since $1 \leqq\left(\right.$ degree $\left.F^{(s)}\right) \leqq n-1, F^{(s)}$ is normal. Of course, the degree of $F^{(s)}$ is regular. Hence, by $\S 42$, there exists an asymptotically constrained extension $\mathscr{G}_{0}$ of $\mathscr{F}_{0}$ in which $F^{(s)}$ has a factorization into linear factors. Then $y_{1} \in \mathscr{G}_{0}$. Hence $\mathscr{G}_{0}$ contains the coefficients of $G$. Thus $G$ is asymptotically inscribed. Therefore, since $G$ is not abnormal, $G$ is normal. Since $G$ is normal, there exists an asymptotically constrained extension $\mathscr{H}_{0}$ of $\mathscr{G}_{0}$ in which $G$ has a root $z_{1}$. Then $y_{1}+z_{1}$ is a root of $F$. But $y_{1}+z_{1} \in \mathscr{H}_{0}$. Thus $\mathscr{H}_{0}$ is an asymptotically constrained extension of $\mathscr{F}_{0}$ containing a root of $F$. Therefore $F$ is normal. This contradiction establishes the lemma.

44. Definition. Let $X$ be loosely closed. Let $n$ be regular for $X$. Let $F$ be an abnormal polynomial of degree $n$. (Cf. Appendix, Note 9.) Let $p$ be a point of instability for $F$. Let $k=$ inst $(p, F)$. Let $\eta$ be the unique root of $F^{(k-1)}$ such that $\eta \sim p$. (We note that inst $\left(p, F^{(k-1)}\right)=1$, by $\$ 21$. Hence, by $(6.2)$, the loose closure of $X$ implies the existence of $\eta$. The uniqueness of $\eta$ is implied by (26.2).) Then

(44.1) the ordered quadruple $(F, p, k, \eta)$ will be called an instability quadruple.

(44.2) If $(F, p, k, \eta)$ and $(G, q, h, \zeta)$ are instability quadruples, then $(G, q, h, \zeta)$ will be called a descendant of $(F, p, k, \eta)$ if $G(z)=F(\eta+z)$, and $q \prec p$.

(44.3) If $Q=\left(Q_{1}, Q_{2}, \ldots, Q_{s}\right)$ is a finite sequence of instability quadruples, with $Q_{j}=\left(F_{j}, p_{j}, k_{j}, y_{j}\right)$, we shall say that $Q$ is a reduction sequence for $Q_{1}$ if $Q_{j+1}$ is a descendant of $Q_{j}(j=1,2, \ldots, s-1)$, and $k_{s}=1$.

45. LEMma. Let $(F, p, k, \eta)$ be an instability quadruple. Let $k=1$. Then $(F, p, k, \eta)$ has no descendants. 
Proof. Since $k=1, F(\eta)=0$. Hence if $G(z)=F(\eta+z)$, then $G(0)=0$. Hence $G$ is not abnormal. Hence $F$ has no descendants.

46. Lemma. Let $Q_{1}=\left(F_{1}, p_{1}, k_{1}, \eta_{1}\right)$ be an instability quadruple. Then the number of distinct reduction sequences for $Q_{1}$ is equal to $k_{1}$.

Proof. If $k_{1}=1$, then, by $\$ 45, Q_{1}$ has no descendants. Hence the only reduction sequence for $Q_{1}$ is the one-term sequence $\left(Q_{1}\right)$. This establishes the result for the case $k_{1}=1$.

Suppose that $r$ is an integer $\geqq 2$ that the conclusion holds whenever $k_{1} \leqq r-1$. Let $Q_{1}=\left(F_{1}, p_{1}, k_{1}, \eta_{1}\right)$ be an instability quadruple with $k_{1}=r$. Let $F_{2}(z)=$ $F_{1}\left(\eta_{1}+z\right)$. Then, by $\S 43, F_{2}$ is abnormal. This implies that $F_{2}(0) \neq 0$. Let $\left\{q_{1}\right.$, $\left.q_{2}, \ldots, q_{m}\right\}$ be the set of those points of instability for $F_{2}$ which satisfy $q_{i} \prec p$. Let $h_{i}=$ inst $\left(q_{i}, F_{2}\right)$. For each $i$ let $\zeta_{i}$ be the root of $F_{2}{ }^{\left(h_{i}-1\right)}$ such that $\zeta_{i} \sim q_{i}$, and let $Q_{2 i}=\left(F_{2}, q_{i}, h_{i}, \zeta_{i}\right)$. Then $S=\left\{Q_{21}, Q_{22}, \ldots, Q_{2 m}\right\}$ is the set of all descendants of $Q_{1}$. Hence $\left(Q_{1}, Q_{2}, \ldots, Q_{s}\right)$ is a reduction sequence for $Q_{1}$ if and only if $Q_{2} \in S$ and $\left(Q_{2}, \ldots, Q_{s}\right)$ is a reduction sequence for $Q_{2}$. By (38.1), $h_{i} \leqq r-1$, and therefore it follows from the original assumption about $r$ that there are $h_{i}$ distinct reduction sequences for $Q_{2 i}$. Since each reduction sequence for $Q_{2 i}$ is distinct from each reduction sequence for $Q_{2 j}$ if $i \neq j$, it follows that the number of distinct reduction sequences for $Q_{1}$ is $\sum_{i=1}^{m} h_{i}$. By (38.2), this sum has the value $k_{1}$. This shows that the conclusion holds for $k_{1}=r$, and establishes the lemma by induction with respect to $k_{1}$.

47. Lemma. Let $Q_{1}=\left(F_{1}, p_{1}, k_{1}, \eta_{1}\right)$ be an instability quadruple. For each reduction sequence $Q=\left(Q_{1}, \ldots, Q_{s}\right)$ for $Q_{1}$, with $Q_{i}=\left(F_{i}, p_{i}, k_{i}, \eta_{i}\right)$, let $y(Q)$ $=\eta_{1}+\eta_{2}+\ldots+\eta_{s}$. Then

(47.1) $y(Q)$ is a root of $F_{1}$.

(47.2) $y(Q) \sim p_{1}$.

(47.3) If $Q$ and $Q^{*}$ are reduction sequences for $Q_{1}$, and $Q \neq Q^{*}$, then $y(Q)$ $\neq y\left(Q^{*}\right)$.

Proof of (47.1). $\quad F_{1}\left(\eta_{1}+\eta_{2}+\ldots+\eta_{s}\right)=F_{2}\left(\eta_{2}+\ldots+\eta_{s}\right)=\ldots=F_{s}\left(\eta_{s}\right)$ $=0$, since $k_{s}=1$.

Proof of (47.2). $\quad \eta_{1} \sim p_{1}$, while $\eta_{2} \sim p_{2} \prec p_{1}$, and likewise $\eta_{i} \prec p_{1}$ for all $i \geqq 2$. Hence $y(Q) \sim p_{1}$.

Proof of (47.3). Let $Q^{*}=\left(Q_{1}{ }^{*}, \ldots, Q_{t}{ }^{*}\right)$, with $Q_{i}{ }^{*}=\left(F_{i}{ }^{*}, p_{i}{ }^{*}, k_{i}{ }^{*}, \eta_{i}{ }^{*}\right)$. We may assume that $s \leqq t$. There must exist $i \leqq s$ such that $Q_{i} \neq Q_{i}{ }^{*}$. (For if $Q_{i}=Q_{i}{ }^{*}$ for all $i \leqq s$, then $k_{s}^{*}=k_{s}=1$, so $Q_{s}{ }^{*}$ has no descendants, and therefore $t=s$, whence $Q^{*}=Q$.) Let $j=\min \left\{i: Q_{i} \neq Q_{i}{ }^{*}\right\}$. Then $j \geqq 2$. Now $Q_{j-1}{ }^{*}=Q_{j-1}$ $=\left(F_{j-1}, p_{j-1}, k_{j-1}, \eta_{j-1}\right)$, and therefore $F_{j}^{*}(z)=F_{j-1}\left(\eta_{j-1}+z\right)=F_{j}(z)$. If $p_{j}^{*}=p_{j}$, then by definition of instability quadruple we have $k_{j}^{*}=k_{j}, \eta_{j}{ }^{*}=\eta_{j}$, and therefore $Q_{j}=Q_{j}{ }^{*}$, which is a contradiction. Hence $p_{j}{ }^{*} \neq p_{j}$.

Let $\sigma=\eta_{1}+\eta_{2}+\ldots+\eta_{j-1}$. Then $y(Q)-\sigma=\eta_{j}+\ldots+\eta_{s} \sim p_{j}$, while 
$y\left(Q^{*}\right)-\sigma=\eta_{j}{ }^{*}+\ldots+\eta_{t}{ }^{*} \sim p_{j}{ }^{*}$ which is not $\sim p_{j}$ (cf. (14.3)). Hence $y(Q)-\sigma$ $\neq y\left(Q^{*}\right)-\sigma$, and therefore $y(Q) \neq y\left(Q^{*}\right)$.

48. Lemma. Let $X$ be loosely closed. Let $n$ be regular for $X$. Let $F$ be abnormal, of degree $n$. Then

(48.1) $F(y)=c\left(y-y_{1}\right)\left(y-y_{2}\right) \ldots\left(y-y_{n}\right)$, with $\left\{y_{1}, \ldots, y_{n}\right\} \subset \mathscr{A}$.

Proof. According to $\S \S 47,46$, and $37, F$ has at least $n$ (hence exactly $n$ ) distinct roots $y_{1}, \ldots, y_{n}$ in $\mathscr{A}$. (We note that $F$ 's abnormality implies that $F(0) \neq 0$.) Therefore we have a factorization (48.1) for some $c$ in $\mathscr{K}_{0}$.

49. Lemma. Let $X$ be loosely closed. Then every asymptotically inscribed polynomial of degree $\geqq 1$ is normal.

Proof. Assume the contrary. Let

$$
n=\min \{s: \exists \text { abnormal polynomial of degree } s\} \text {. }
$$

By $\$ 40, n \geqq 2$. Obviously, $n$ is regular. Let $F$ be an abnormal polynomial of degree $n$. Let $\mathscr{F}_{0}$ be an asymptotically constrained field containing the coefficients of $F$. Let $y_{1}$ be a root of $F$ in $\mathscr{A}$ (cf. $\$ 48$ ). Let $\mathscr{G}_{0}=\mathscr{F}_{0}\left(y_{1}\right)$. Now if $h$ is any element of $\mathscr{G}_{0}, h$ is a root of a polynomial $G$, with coefficients in $\mathscr{F}_{0}$, such that the degree $d$ of $G$ satisfies $1 \leqq d \leqq n$. Then $G$ is asymptotically inscribed. By $\S \S 42$ and 48 , we see that $h \in \mathscr{A}_{0}$. Hence $\mathscr{G}_{0} \subset \mathscr{A}_{0}$. Thus $\mathscr{G}_{0}$ is an asymptotically constrained extension of $\mathscr{F}_{0}$ containing a root $y_{1}$ of $F$. Hence $F$ is normal. This contradiction proves the Lemma.

50. Proof of Theorem I, $\S 8$.

Proof of (8.1). Let $X$ be loosely closed. Let $\mathscr{F}_{0}$ be a field asymptotically constrained over $X$. By Zorn's Lemma, there exists a maximal (in the sense of inclusion) asymptotically constrained field $\mathscr{J}_{0}$ containing $\mathscr{F}_{0}$. By $\S 49, \mathscr{J}_{0}$ is algebraically closed.

Proof of (8.2). Let $X$ be tightly closed. Let $F(y)=\sum a_{i} y^{i}$ be any polynomial of degree $\geqq 1$ with coefficients in $\mathscr{C}_{0}$. We may and do assume that $F(0) \neq 0$. Let $y_{0}$ be a root of $F$, with $y_{0}$ in $\mathscr{A}_{0}$. Then $y_{0} \in \mathscr{A}$. Let $y_{0} \sim p \in \mathscr{M}$. Then, by $\S 26, p$ is a point of instability for $F$. Hence, by $\S 36, p$ is an approximate zero for $F$. Let $u=] p[$, and let $\zeta=p / u$. Let $(r, s, u)$ be a Newton triple for $F$. Then $a_{r} u^{r} \approx a_{s} u^{s}$, whence $u^{r-s} \approx 1$, which by (16.3) and (2.2) implies $u=1$. Hence $p=\zeta \in \mathscr{C}$. Let $y_{0}=p+e$. Then $e \prec 1$. We have $0=F\left(y_{0}\right)=F(p)+\varepsilon$, where $\varepsilon=\sum_{j=1}^{n} e^{j} F^{(j)}(p)(j !)^{-1}$. Evidently $\varepsilon \prec 1$. Hence $F(p) \prec 1$. But $F(p) \in \mathscr{C}_{0}$. Hence, by (14.1), $F(p)=0$. Thus $F$ has a root $p$ in $\mathscr{C}$. Hence $\mathscr{C}_{0}$ is algebraically closed. This gives (6.1).

Now let $F$ be asymptotically inscribed over $X$, and let $w$ be a point of instability for $F$. Let $\mathscr{F}_{0}$ be an asymptotically constrained field containing the coefficients of $F$. Let $\mathscr{G}_{0}$ be an asymptotically constrained field which is algebraically 
closed and includes $\mathscr{F}_{0}$. Then $F=c\left(y-y_{1}\right) \ldots\left(y-y_{n}\right)$ with $\left\{c, y_{1}, \ldots, y_{n}\right\} \subset \mathscr{G}_{0}$. If no $y_{i}$ is $\sim w$, it follows from repeated applications of $\$ \S 24$ and 25 that inst $(w, F)$ $=0$. This contradiction gives the result that for every point of instability $w$ there is a root $y_{0}$ of $F$ such that $y_{0} \sim w$. In particular, it gives (6.2).

\section{Part II. Fields OF Meromorphic Functions}

51. Definition. By a domain system will be meant a filter base $N$ each element of which is a region in the finite complex plane. (Cf. Appendix, Note 10.)

52. Definition. Let $\bar{N}$ be a domain system. Then the function $\phi$ will be called analytic over $\bar{N}$ if the domain of $\phi$ includes at least one element of $\bar{N}$, and $\phi$ is analytic at every point of the domain of $\phi$. (Cf. Appendix, Note 11.) The concept meromorphic over $\bar{N}$ is defined in a similar manner.

53. Notation. Let $\bar{N}$ be a domain system. Then $\widetilde{K}_{0}(\bar{N})=\{\phi: \phi$ meromorphic over $\bar{N}\}$.

54. Definition. If $\bar{N}$ is a domain system, and $\{\phi, \gamma\} \subset \tilde{K}_{0}(\bar{N})$, we shall say that $\phi=\gamma($ over $\bar{N})$ if there exists an element $W$ of $\bar{N}$ such that $\phi(x)=\gamma(x)$ for all $x$ in $W$.

55. Definition. Let $\bar{N}$ be a domain system. Let $\sigma \in \mathbf{C}$. Let $\phi \in \widetilde{K}_{0}(\bar{N})$. Then we write $\phi \rightarrow \sigma$ (over $\bar{N}$ ) if for every $\varepsilon>0$ there exists $W \in \bar{N}$ such that $|\phi(x)-\sigma|<\varepsilon$ for all $x \in W$. (Cf. Appendix, Note 12.)

56. Definition. Let $\bar{N}$ be a domain system. Let $\tilde{E}_{0}$ be a subset of $\tilde{K}_{0}(\bar{N})$. Then $\tilde{E}_{0}$ will be called a subconstant class over $\bar{N}$, and the ordered pair $\left(\bar{N}, \tilde{E}_{0}\right)$ will be called a subconstant pair, if all the following conditions are satisfied:

(56.1) If $\phi \in \tilde{E}_{0}$, and $\gamma=\phi(\operatorname{over} N)$, then $\gamma \in \tilde{E}_{0}$.

(56.2) If $\phi \in \tilde{E}_{0}$, then $\phi \rightarrow 0$ (over $\bar{N}$ ).

(56.3) If $\phi \in \tilde{E}_{0}$, and $\gamma \in \widetilde{E}_{0}$, then $\phi \gamma \in \widetilde{E}_{0}$, and $\phi-\gamma \in \widetilde{E}_{0}$ and $(1+\gamma)^{-1} \phi \in \widetilde{E}_{0}$.

(56.4) If $\phi \in \tilde{E}_{0}$, and $\sigma \in \mathbf{C}$, then $\sigma \phi \in \tilde{E}_{0}$.

57. Definition. Let $\left(\bar{N}, \tilde{E}_{0}\right)$ be a subconstant pair. Then $\left(\bar{N}, \tilde{E}_{0}\right)$ is called quasi-linearly closed if for every non-negative integer $n$ and every ordered $(n+1)$ tuple $\left(\varepsilon_{0}, \ldots, \varepsilon_{n}\right)$ of elements of $\tilde{E}_{0}$ there exists an element $\eta$ of $\tilde{E}_{0}$ such that $\eta=\sum_{j=0}^{n} \varepsilon_{j} \eta^{j}$.

58. Definition. Let $\left(\bar{N}, \tilde{E}_{0}\right)$ be a subconstant pair. Let $\{\phi, \gamma, \zeta\} \subset \widetilde{K}_{0}(\bar{N})$. We write $\phi \prec \gamma\left(\operatorname{over}\left(\bar{N}, \widetilde{E}_{0}\right)\right)$ if $\gamma \neq 0$ and $\phi \gamma^{-1} \in \tilde{E}_{0}$, and we write $\zeta \sim \gamma$ (over $\left.\left(\bar{N}, \tilde{E}_{0}\right)\right)$ if $\zeta-\gamma \prec \gamma\left(\operatorname{over}\left(\bar{N}, \tilde{E}_{0}\right)\right)$.

59. Notation. Let $\tilde{V}$ be a subset of the real numbers, such that $\tilde{V}$ is a vector space over the field of rational numbers. We shall call $\tilde{V}$ a real // rational vector space.

60. Definition. Let $\left(\bar{N}, \widetilde{E}_{0}\right)$ be a subconstant pair. Let $\tilde{V}$ be a real//rational vector space. Let $\tilde{L}$ be a nonempty set of functions analytic over $\bar{N}$. We shall say that $\left(\bar{N}, \tilde{E}_{0}, \tilde{V}, \tilde{L}\right)$ is a logarithmic quadruple if 
(60.1) Whenever $\phi \in \tilde{L}$ and $\gamma \in \tilde{L}$, then either $\phi=\gamma$, or $\exp \phi \prec \exp \gamma$, or $\exp \gamma$ $\prec \exp \phi$.

(60.2) Whenever $\phi \in \tilde{L}$ and $\gamma \in \tilde{L}$, and $\exp \phi \prec \exp \gamma$, then $\exp (\sigma \phi) \prec \exp (\tau \gamma)$ for all (strictly) positive $\sigma$ and $\tau$ in $\tilde{V}$.

61. Definition. Let $Y=\left(\bar{N}, \tilde{E}_{0}, \tilde{V}, \tilde{L}\right)$ be a logarithmic quadruple. Then

(61.1) Every function meromorphic over $\bar{N}$ which has, in some element of $\bar{N}$, a representation of the form $\exp \left(v_{1} \lambda_{1}+v_{2} \lambda_{2}+\ldots+v_{s} \lambda_{s}\right)$ with $v_{i} \in \tilde{V}$ and $\lambda_{i} \in \tilde{L}$ will be called a unit Y-monomial. The set of all unit $Y$-monomials will be denoted by $\tilde{U}(Y)$.

(61.2) Every function of the form $c u$, where $c \in \mathbf{C}-\{0\}$ and $u \in \tilde{U}(Y)$ will be called a $Y$-monomial. The set of all $Y$-monomials will be denoted by $\tilde{M}(Y)$.

(61.3) Every function $h$ for which there exists an element $g$ of $\tilde{M}(Y)$ such that $h \sim g$ will be called an asymptotic $Y$-monomial, and the set of all asymptotic $Y$-monomials will be denoted by $\tilde{A}(Y)$.

(61.4) Every field (cf. Appendix, Note 5) $\tilde{F}_{0}$ such that $\tilde{M}(Y) \subset \tilde{F}_{0} \subset \tilde{A}(Y) \cup\{0\}$ will be called a $Y$-field.

62. TheOREM II. Let $Y=\left(\bar{N}, \tilde{E}_{0}, \tilde{V}, \tilde{L}\right)$ be a logarithmic quadruple. Let $\left(\bar{N}, \widetilde{E}_{0}\right)$ be quasi-linearly closed. Then if $\tilde{F}_{0}$ is any $Y$-field, there exists an extension $\tilde{G}_{0}$ of $\tilde{F}_{0}$ such that $\tilde{G}_{0}$ is a $Y$-field and is algebraically closed.

Proof. The proof is given in $\$ 65$.

63. Notation. If $\bar{N}$ is a domain system, and $\phi \in \tilde{K}_{0}(\bar{N})$, the symbol $\phi \#$ will represent the set $\left\{\gamma: \gamma \in \tilde{K}_{0}(\bar{N}) ; \gamma=\phi(\right.$ over $\left.\bar{N})\right\}$. If $\tilde{H} \subset \widetilde{K}_{0}(\bar{N}), \tilde{H} \#$ will represent the set $\{\zeta \#: \zeta \in \tilde{H}\}$.

64. Fundamental Lemma B. Let $Y=\left(\bar{N}, \widetilde{E}_{0}, \tilde{V}, \tilde{L}\right)$ be a logarithmic quadruple. Let $\mathscr{K}_{0}=\left(\tilde{K}_{0}\right) \#$, and for $\{\phi \#, \gamma \#\} \subset \mathscr{K}_{0}$, let the following definitions be made:

$$
\begin{aligned}
& \phi \#+\gamma \#=(\phi+\gamma) \#, \\
& \phi \# \gamma \#=(\phi \gamma) \#, \\
& \phi \#<\gamma \# \text { iff } \phi \prec \gamma .
\end{aligned}
$$

Let $\mathscr{U}=(U(Y)) \#$. Let $\mathscr{C}_{0}=\mathbf{C} \#$. Then

(64.1) $\left(\mathscr{K}_{0}, \prec, \mathscr{U}, \mathscr{C}_{0}\right)$ is a graduated field.

(64.2) If $\left(\bar{N}, \widetilde{E}_{0}\right)$ is quasi-linearly closed, then $\left(\mathscr{K}_{0}, \prec, \mathscr{U}, \mathscr{C}_{0}\right)$ is loosely closed.

Proof of (64.1). This is verified in a completely straightforward fashion.

Proof of (64.2). It suffices to verify (6.2). We assert first that if $\mathscr{E}_{0}=\left(\widetilde{E}_{0}\right) \#$, then for every non-negative integer $n$ and every ordered $(n+1)$-tuple $\left(e_{0}, \ldots, e_{n}\right)$ of elements of $\mathscr{E}_{0}$ there exists an element $z$ of $\mathscr{E}_{0}$ such that $z=\sum_{i=0}^{n} e_{i} z^{i}$. To establish this assertion we note that by definition of $\mathscr{E}_{0}$ there exists an ordered $(n+1)$-tuple $\left(\varepsilon_{0}, \ldots, \varepsilon_{n}\right)$ of elements of $E_{0}$ such that $e_{i}=\varepsilon_{i} \#(i=0, \ldots, n)$. Then, since $\left(\bar{N}, \tilde{E}_{0}\right)$ is quasi-linearly closed, there exists $\zeta$ in $\tilde{E}_{0}$ such that 
$\zeta=\sum_{i=0}^{n} \varepsilon_{i} \zeta^{i}$. Then if $z=\zeta \#$, we have $z=\sum_{i=0}^{n} e_{i} z^{i}$, and $z \in \mathscr{E}_{0}$, which establishes the assertion.

Now let $\mathscr{F}_{0}$ be an asymptotically constrained subfield of $\mathscr{K}_{0}$. Let $F(y)$ $=\sum_{i=0}^{n} f_{i} y^{i}$, with $f_{i} \in \mathscr{F}_{0}$, and let us assume, as we may without essential loss of generality, that $f_{0} f_{n} \neq 0$. Let inst $(w, F)=1$.

By $\S 36, \operatorname{azm}(w, F)=1$. Let $m=] w[$. Let $t=w / m$. Let $(r, s, m, D)$ be a Newton quadruple for $F$. Let $G(z)=\left(f_{r} m^{r}\right)^{-1} F(m z)=\sum^{n}=0 g_{i} z^{i}$. Then $g_{i} \in \mathscr{F}_{0}$, and $g_{i} \lesssim 1$, and if $d_{i}=\operatorname{proj}\left(g_{i}\right)$, we have $D(z)=\sum_{i=0}^{n} d_{i} z^{i}$. Now $D(t)=0 \neq D^{\prime}(t)$, so $G(t) \prec 1$ and $G^{\prime}(t) \approx 1$.

Let $Q(u)=G(t+u)=\sum_{i=0}^{n} q_{i} u^{i}$. Then $q_{i} \in \mathscr{F}_{0}$. Also, since $q_{i}=(i !)^{-1} G^{(i)}(t)$, we have $q_{0} \prec 1, q_{1} \approx 1, q_{j} \precsim 1$.

If $q_{0}=0$, then $Q(0)=0$, so $G(t)=0$, and therefore $F(w)=0$. In this case we may take $y_{0}=w$.

If $q_{0} \neq 0$, let $\left.b=\right] q_{0}\left[\right.$. Then $b \in \mathscr{U}$ and $b \prec 1$. Let $h=b^{1 / 2}$. Then $q_{0} \prec h \prec 1$. The equation $Q(u)=0$ is transformed by the substitution $u=h v$, followed by division by $q_{1} h$, into an equation $R(v)=0$, where $R(v)=v-\sum_{i=0}^{n} e_{i} v^{i}$, with $e_{0}=-q_{0} q_{1}^{-1} h^{-1} \prec 1, e_{1}=0, e_{i}=-q_{i} q_{1}^{-1} h^{i-1} \prec 1(i \geqq 2)$. By the assertion in the first paragraph of the proof of (64.2) there exists $v_{0} \prec 1$ such that $R\left(v_{0}\right)=0$. Then if $y_{0}=w\left(t+h v_{0}\right)$, we have $y_{0} \sim w$, and $F\left(y_{0}\right)=0$. This completes the verification of (6.2).

65. Proof of Theorem II. This is a corollary of Fundamental Lemma B (in §64) and Theorem I (in §8).

\section{PART III. EXAmples for PART II}

66. Preliminary notations.

(66.1) Let $\gamma$ satisfy $0<\gamma \leqq \pi$. Let $r$ be a non-negative real number. Then by $S(r, \gamma)$ will be meant the sector $\{x: x \in \mathbf{C} ;|x| \geqq r ;|\arg x|<\gamma\}$.

(66.2) The functions $\log x, \log \log x, \ldots, \log _{p} x, \ldots$ are (for the purposes of this paper) determined by the stipulations that domain $\left(\log _{p} x\right)=S\left(e_{p-1}(0), \pi\right)$ (where $e_{0}(x)=x$ and $\left.e_{j+1}=\exp \left(e_{j}\right)\right)$, and that $\log _{p} x>0$ for all $x$ such that $x>e_{p}(0)$.

(66.3) Let $\tilde{V}$ be any real// rational vector space. The function $f$ will be called a $(\tilde{V}, p)$-logarithmic monomial (or briefly, a logarithmic monomial), if there exists a nonzero complex number $c$ and an ordered $(p+2)$-tuple $\left(r_{0}, r_{1}, \ldots, r_{p+1}\right)$ in $\tilde{V}$ such that $f(x)=c \exp \left(r_{0} \cdot 0+r_{1} \log x+r_{2} \log \log x+\ldots+r_{p+1} \log _{p+1} x\right)$ (which is also written as $c x^{r_{1}}(\log x)^{r_{2}} \ldots\left(\log _{p} x\right)^{r_{p}+1}$.) The set of all $(\tilde{V}, p)$-logarithmic monomials will be denoted by $L \cdot M(\tilde{V}, p)$.

67. EXAMPLES OF DOMAIN SYSTEMS. For each $\gamma$ such that $0<\gamma \leqq \pi$, let $\bar{N}_{1}(\gamma)$ $=\{S(r, \gamma): r \geqq 0\}$. For each $r \geqq 0$, let $\bar{N}_{2}(r)=\{S(r, \gamma): 0<\gamma \leqq \pi\}$. Then each $\bar{N}_{1}(\gamma)$ is a domain system, and each $\bar{N}_{2}(r)$ is a domain system.

68. Definition. Let $\bar{N}$ be any domain system which has the following two properties: 
(68.1) No element of $\bar{N}$ meets the nonpositive real axis.

(68.2) $\lim \bar{N}=\infty$, i.e., for every $G>0$ there exists an element $A$ of $\bar{N}$ such that $|x|>G$ whenever $x \in A$.

As in [A]§3, (cf. Appendix, Note 13), we shall call such an $\bar{N}$ a complex neighborhood system of $+\infty$, or, briefly, a $C S$. We note that in $\S 67$ each $\bar{N}_{1}(\gamma)$ is a $\bar{C} S$, while no $\bar{N}_{2}(r)$ is a $\bar{C} S$.

It is obvious that if $N$ is any $C S$, then all the functions $\log x, \log \log x, \ldots$ are analytic over $\vec{N}$.

69. EXAMPLES OF SUBCONSTANT PAIRS.

(69.1) Let $\vec{N}$ be any domain system. Let $q$ be a non-negative integer. Let $\tilde{E}_{0}(q, \bar{N})=\left\{f: f\right.$ analytic over $\bar{N} ; f^{(j)} \rightarrow 0$ over $\left.\bar{N}(j=0,1, \ldots, q)\right\}$. Let $\widetilde{E}_{0}(\infty, \bar{N})$ $=\left\{f: f\right.$ analytic over $\bar{N} ; f^{(j)} \rightarrow 0$ over $\bar{N}($ all $\left.j)\right\}$. Then $\left(\bar{N}, \tilde{E}_{0}(q, \bar{N})\right)$ is a subconstant pair, and so is $\left(\bar{N}, \tilde{E}_{0}(\infty, \bar{N})\right)$. It follows readily from the implicit function theorem that all these subconstant pairs are quasi-linearly closed.

(69.2) Let $\bar{N}$ be a $\bar{C}$. A subconstant class $\widetilde{E}_{0} *(\bar{N})$ over $\bar{N}$ can be defined in the following way: $f \in \widetilde{E}_{0} *(\bar{N})$ iff for every ordered pair of non-negative integers $(j, p)$ we have $\left(x \log x \log \log x \ldots \log _{p} x d / d x\right)^{j} f \rightarrow 0$ over $\bar{N}$. With this definiton of $\tilde{E}_{0}$ the order relation $\prec$ becomes the one used in $[\mathrm{A}]$ and $[\mathrm{P}]$ for the study of differential equations, where it possesses certain crucial properties in its behavior under differentiation (e.g. if $f \prec g \sim h$, where $h$ is a nonconstant logarithmic monomial, then $\left.f^{\prime} \prec g^{\prime}\right)$. It is easy to see that $\left(\bar{N}, \widetilde{E}_{0} *(\bar{N})\right)$ is quasi-linearly closed. (Cf. [A], §36.)

(69.3) Let $\bar{N}$ be a $\bar{C} S$. Let $\tilde{V}$ be any real//rational vector space. Let $p$ be an integer $\geqq 0$. Let $\tilde{E}_{p 0}(\bar{N}, \tilde{V})$ be the set of functions $f$ defined as follows: $f \in \widetilde{E}_{p 0}(\bar{N}, \tilde{V})$ if there exists an integer $s \geqq 1$ and a function $G\left(x_{1}, x_{2}, \ldots, x_{s}\right)$ of $s$ complex variables, analytic and vanishing at the origin, and an ordered $s$-tuple $\left(\varepsilon_{1}, \varepsilon_{2}, \ldots, \varepsilon_{s}\right)$ of elements of $L \cdot M(\tilde{V}, p)$ with $\varepsilon_{i} \rightarrow 0$ over $\bar{N}$, such that, in some element of $\bar{N}, f$ has a representation $f=G\left(\varepsilon_{1}, \varepsilon_{2}, \ldots, \varepsilon_{s}\right)$. It is easy to see, by the implicit function theorem, that $\left(\bar{N}, \widetilde{E}_{p 0}(\bar{N}, \tilde{V})\right)$ is a quasi-linearly closed subconstant pair.

70. EXAMPLES OF LOGARITHMIC QUADRUPLES. Let $\bar{N}$ be any $\bar{C} S$. Let $\tilde{V}$ be any real//rational vector space. Let $p$ be any integer such that $p \geqq 0$. Let $\widetilde{S}_{p}$ $=\left\{0, \log x, \log \log x, \ldots, \log _{p+1} x\right\}$. Then if $\tilde{E}_{0}$ is chosen as any one of the following subconstant classes over $\bar{N}: \tilde{E}_{0}(q, \bar{N}), \tilde{E}_{0}(\infty, \bar{N}), \tilde{E}_{0} *(\bar{N}), \tilde{E}_{p 0}(\bar{N}, \tilde{V})$, the ordered quadruple $Y=\left(\tilde{N}, \tilde{E}_{0}, \tilde{V}, \tilde{S}_{p}\right)$ will be a logarithmic quadruple.

We note that in all these cases the set $\tilde{M}(Y)$ (in the notation of (61.2)), that is, the set such that $\tilde{M}(Y) \#$ is the group $\mathscr{M}$ of the corresponding graduated field (cf. $\S \S 63,64)$ is a set of logarithmic monomials. (Cf. Appendix, Note 14.)

71. EXAMPLES OF THE APPLICATION OF THEOREM II.

(71.1) If we apply Theorem II to the logarithmic quadruple $\left(\bar{N}, \tilde{E}_{0}(q, \vec{N}), \mathbf{R}, \widetilde{S}_{p}\right)$ we get this result:

Let $\tilde{F}_{0}$ be a field of functions which includes $L \cdot M(R, p)$ and which has the 
following property: For every nonzero element $g$ of $\tilde{F}_{0}$ there exists an element $h$ of $L \cdot M(\mathbf{R}, p)$ such that $g \sim h$ in the sense that $D^{j}\left(g^{-1} h-1\right) \rightarrow 0$ over $\bar{N}$ $(j=0,1, \ldots, q)$.

Then every polynomial $P$ of degree $n \geqq 1$ with coefficients in $\tilde{F}_{0}$ will have $n$ roots $y_{1}, \ldots, y_{n}$ each of which is $\sim$ (in the same sense) to an element of $L \cdot M(\mathbf{R}, p)$.

(71.2) If we replace, in $(71.1), \tilde{E}_{0}(q, \bar{N})$ by $\widetilde{E}_{0} *(\bar{N})$, we get a corresponding result in which the coefficients of the polynomial $P$, and the roots $y_{1}, \ldots, y_{n}$, are asymptotically equivalent to logarithmic monomials in a much more stringent sense (cf. Appendix, Note 15). This result is very useful in the study of differential equations. (Cf. Part IV below.)

(71.3) If we choose the logarithmic quadruple $Y=\left(\bar{N}, \tilde{E}_{p o}(\tilde{V}, \bar{N}), \tilde{V}, \tilde{S}_{p}\right)$, with $\bar{N}$ any $\overline{C S}$, and $\tilde{V}$ any real// rational vector space, we find that (in the terminology of (61.3)), $\tilde{A}(Y) \cup\{0\}$ is identical with the totality $\widetilde{B}_{p o}(\tilde{V}, \bar{N})$ of all functions having, in an element of $\bar{N}$, a representation $m G\left(\varepsilon_{1}, \varepsilon_{2}, \ldots, \varepsilon_{s}\right)$, where $G$ is analytic at $(0,0, \ldots, 0)$ and $m, \varepsilon_{1}, \ldots, \varepsilon_{s}$ are elements of $L \cdot M(\tilde{V}, p)$ such that $\varepsilon_{j} \rightarrow 0$ over $\bar{N}$. (Cf. Appendix, Note 16.) From this it is easy to see that $\tilde{A}_{0}(Y)$ is a field. Then it follows at once from Theorem II that $\tilde{A}_{0}(Y)$ is algebraically closed.

(71.4) If in the result of (71.3) we specialize $p$ to 0 and $\tilde{V}$ to $\mathbf{Q}$ we get the result that the field of all functions $f$ having, in an element of $\bar{N}$, a representation of the form (*) $f(x)=x^{p / q} \sum_{i=0}^{\infty} c_{i} x^{i / q}$ (where $p$ and $q$ are integers, with $q \leqq-1$ ) is algebraically closed. This gives in particular the classic theorem on the expansion of an algebraic function of one variable in the neighborhood of a branch point. (The branch point here is taken at $\infty$; if we take $\bar{N}=\bar{N}_{1}(\gamma)$ (for any $\gamma$ such that $0<\gamma \leqq \pi)$ the sectorial region of validity of the expansions (*) can obviously, in the case of algebraic functions, be extended by analytic continuation to a neighborhood of $\infty$ on an appropriate Riemann surface.)

It might be remarked that the problems solved in (71.1) and (71.2) are fundamentally more dificult than those solved in (71.3) and (71.4), precisely because in (71.1) and (71.2) $\tilde{A}_{0}(Y)$ is not a field: asymptotic equivalence (to logarithmic monomials) as defined by means of $E_{0}(q, \bar{N})$ or $E_{0} *(\bar{N})$ is not so stringently specified that it survives, in general, under the operation of addition.

\section{Part IV. Applications to Algebraic Differential Equations}

In the terminology of $\S 67$, let $\bar{N}=\bar{N}_{1}(\gamma)$ for some $\gamma$ such that $0<\gamma \leqq \pi$.

We consider first-order algebraic differential equations $F\left(x, y, y^{\prime}\right)$ $=\sum_{i, j=0}^{n} f_{i j}(x) y^{i}\left(y^{\prime}\right)^{j}=0$, with $f_{00} \neq 0$, and with the $f_{i j}$ belonging to a set of functions $\tilde{G}_{0}$ which is a logarithmic differential field of logarithmic rank $p$ over $\bar{N}$, (briefly, an $\operatorname{LDF}_{p}(\bar{N})$ ). (This means that $\tilde{G}_{0}$ is a $Y$-field, in the terminology of (61.4), with $Y=\left(\bar{N}, \tilde{E}_{0} *(\bar{N}), \mathbf{R}, \tilde{S}_{p}\right)$, and has the additional property that if $f \in \tilde{G}_{0}$, then $f^{\prime} \in \widetilde{G}_{0}$.) We seek a solution which is $\sim$ to a logarithmic monomial. 
We define a principal monomial to be a logarithmic monomial $m$ such that $F$ is not stable at $m$, but $F$ is stable at every logarithmic monomial $h$ such that $h \prec m$ (where stable is defined as in §5). The principal monomials constitute a finite set which can be determined by an algorithm which is an elaboration of the Newton polygon algorithm described in $\$ 28$ (cf. [A], §66). It simplifies the discussion to suppose, as we shall, that we are treating the case of a principal monomial equal to 1 . (This case can always be realized by a transformation $y=m z$.) Analogous to the instability multiplicity defined in $\$ 5$ are two indices (corresponding to the two arguments $y^{\prime}, y$ in $F$ ). One index is the singularity multiplicity $s^{*}=\min \left\{k: \partial^{k} F /\left(\partial y^{\prime}\right)^{k}\right.$ is stable at 1$\}$. The other index is the algebraic multiplicity $a^{*}=\min \left\{h: \partial^{h} F /(\partial y)^{h}\right.$ is stable at 1$\}$.

In the case $a^{*}=1$ and $s^{*}=1$, the equation $F=0$ is reduced to a quasi-linear form by the substitution $y=1+z$. Then if subsidiary conditions are fulfilled (e.g., if $\tilde{G}_{0}$ is Schwarzian-symmetric (by which is meant that for every $f$ in $\widetilde{G}_{0}$ the Schwarzian image $g$ of $f$, defined by $g(x)=(f(\bar{x}))^{-}$, is in $\left.\tilde{G}_{0}\right)$ ) a solution $y_{0}$ can be found, with $y_{0} \sim 1$, over $\left(\bar{N}_{1}(\delta), \widetilde{E}_{0} *\left(\bar{N}_{1}(\delta)\right)\right)$, for some $\delta$ satisfying $0<\delta \leqq \gamma$

In the case $s^{*}=1$, and $a^{*}$ arbitrary, we can, using both Theorem II, $\$ 62$, as applied in (71.2), and the method of proof of Fundamental Lemma A, §38, show that by means of substitutions of the form $y=\eta+z$, with $\eta$ a solution of an algebraic equation with coefficients in $\tilde{G}_{0}, a^{*}$ can be reduced to 1 , with $s^{*}$ kept equal to 1 , so that the result of the previous paragraph may be applied to show the existence of a solution $\sim 1$.

In some cases where both $s^{*}$ and $a^{*}$ are arbitrary, similar methods suffice to reduce $s^{*}$ to 1 , after which the above results will apply. This is the case, for instance, with the equation $F\left(x, y, y^{\prime}\right)=\sum_{i=0}^{n} a_{i}(x) y^{i}+\sum_{i=0}^{n} b_{i}(x)\left(y^{\prime}\right)^{i}$, with the set $\left\{a_{0}, \ldots, a_{n}, b_{0}, \ldots, b_{n}\right\}$ included in an $\mathrm{LDF}_{p}$.

These questions, and other problems in the theory of differential equations to which the methods and results of the present paper apply, will be treated in forthcoming papers by the author.

\section{PART V. APPENDIX}

NoTE 1. Let $\mathscr{K}_{0}$ be a commutative field having a non-Archimedean valuation $\phi$ trivial over the prime field $\pi$, (cf. van der Waerden, Modern algebra, Vol. I, pp. 235, 238, Ungar, New York, 1949). If $f<g$ is defined to mean $\phi(f)<\phi(g)$, and $\mathscr{C}_{0}$ is set equal to $\pi$, and $\mathscr{U}$ is set equal to $\{1\}$, then $\left(\mathscr{K}_{0}, \prec, \mathscr{U}, \mathscr{C}_{0}\right)$ will be a graduated field. As a step in the opposite direction we note that if we define, for elements of $\mathscr{A}, \psi(f)=] f[$ (cf. $\S 17$ ), then $f \prec g$ is equivalent, for $\{f, g\}<\mathscr{A}$, to $\psi(f)<\psi(g)$, and $\psi$ has all the properties of a non-Archimedean valuation (with values in the ordered Abelian group $\mathscr{U}$ ), except that its domain is restricted to $\mathscr{A}$, instead of being equal to the field $\mathscr{K}_{0}$. 
Note 2. We use the older term "partial order" in preference to the term "order" since we wish to emphasize the fact that we are not dealing here with an "ordered field" (e.g., $a \prec b, c \prec d$ does not imply $a+c \prec b+d$ ).

Note 3. Subsets of $\mathscr{K}_{0}$ which contain 0 will always be denoted by a letter with a zero subscript, such as $\mathscr{E}_{0}$, and $\mathscr{E}_{0}-\{0\}$ will be denoted by the corresponding symbol $\mathscr{E}$ without the zero subscript. Conversely, subsets of $\mathscr{K}_{0}$ which do not contain 0 will always be denoted by a letter without zero subscript, such as $\mathscr{F}$, and the symbol $\mathscr{F}_{0}$ will be used for $\mathscr{F} \cup\{0\}$.

Note 4. Theorem I asserts the converse as well: (c) implies (a) and (b). This converse statement is by far the easier part of the theorem.

Note 5. Since there is, in general, no common domain for the class $\widetilde{K}_{0}(\bar{N})$ of functions meromorphic over $\bar{N}$, there is a technical difficulty in the interpretation of an individual element $f$ of $\tilde{K}_{0}(\bar{N})$ as an element of a field. What more conveniently plays this role is the class of functions equivalent to $f$ under an appropriate type of analytic continuation. This distinction is observed in the formulation of Fundamental Lemma $B, \S 64$, but, in the interests of notational brevity, is otherwise ignored in this paper.

Note 6. We use the customary notations $\mathbf{C}, \mathbf{R}, \mathbf{Q}$ for the fields of complex numbers, real numbers, and rational numbers, respectively. For each $c$ in $\mathbf{C}$ the function having the constant value $c$ at all points of the finite complex plane will also be denoted by $c$.

NoTE 7. For every graduated field there exist asymptotically constrained fields. The smallest example of such is the field generated by $\mathscr{M}$.

Note 8 . In the sequel, the reference $(x) /(y)$ means the statement obtained by applying Statement $(x)$ to Statement $(y)$. Thus, e.g., $(16.2) /(10.1)$ is the statement "If $f \approx g$ then $f \neq 0$ and $g \neq 0$."

Note 9. In $\$ \S 44-49$ our objective, which is achieved in $\$ 49$, will be to show that in the case where $X$ is loosely closed there are no abnormal polynomials. Thus, in fact, the lemmas of $\$ \S 45-48$ are all vacuous.

NoTE 10 . That is, $\bar{N}$ is a nonempty set of nonempty connected open sets, such that if $\Omega$ and $\Lambda$ are elements of $\bar{N}$, then there exists an element $\Delta$ of $\bar{N}$ such that $\Delta \subset \Omega \cap \Lambda$. (Cf. N. Bourbaki, Topologie générale, Chapter I, §5, no. 4.)

Note 11 . We are not dealing here with the "complete analytic function", in the sense of Weierstrass; it is possible, for example, for a function which is analytic over $\bar{N}$ to have extensions and restrictions which are not analytic over $\bar{N}$.

Note 12. This, of course, is the standard definition of convergence over a filter (N. Bourbaki, Topologie générale, Chapter I, §6, no. 4), as it would carry over to the case of a filter base.

Note 13. References [A] and [P] are to the following papers by the author: [A] Contributions to the asymptotic theory of ordinary differential equations in the complex domain, Mem. Amer. Math. Soc. No. 13 (1954), 81 pp. 
[P] Principal solutions of ordinary differential equations in the complex domain, Mem. Amer. Math. Soc. No. 26 (1957), 107 pp.

NoTE 14. The emphasis in $\$ \$ 70-71$ upon the very special choice of $\tilde{S}_{p}$ for $\tilde{L}$ is dictated by the importance of logarithmic monomials in the theory of algebraic differential equations with rational coefficients.

Note 15. In [P] $\$ 44$ there is asserted obliquely (without proof) a stronger result to the effect that if the coefficients of a polynomial $F(y)=\sum_{i=0}^{n} a_{i} y^{i}$ belong to a "logarithmic domain of logarithmic rank $\leqq p$ " (cf. [A], §49), and $a_{0} a_{n}$ is "non-trivial," then the roots of $F$ will be $\sim$ elements of $L \cdot M(R, p)$ in the strong sense determined by $\tilde{E}_{0} *(\bar{N})$. This stronger assertion is false. The underlying considerations justified only the conclusion that the roots are $\sim$ elements of $L \cdot M(\mathbf{R}, p)$ in the weak sense determined by $\widetilde{E}_{0}(0, \bar{N})$. (Fortunately, the weaker conclusion sufficed for the purpose then at hand, namely the selection of certain logarithmic monomials.) I am indebted to E. W. Chamberlain for drawing my attention to this error in [P].

Note 16. This is easy but not completely trivial. Let $\tilde{A}_{0}(Y)=\tilde{A}(Y) \cup\{0\}$. Since $\tilde{A}(Y)$, by definition, is equal to the set

$$
\left\{m(1+e): m \in L \cdot M(\tilde{V}, p) ; e \in \tilde{E}_{p 0}(\tilde{N}, \tilde{V})\right\}
$$

it is almost obvious that $\tilde{A}_{0}(Y) \subset \widetilde{B}_{p 0}(\tilde{V}, \bar{N})$. Suppose that $f \in \widetilde{B}_{p 0}(\tilde{V}, \bar{N})$. Then $(* *) f=m G\left(\delta_{1}, \ldots, \delta_{s}\right)$ with $\left\{m, \delta_{1}, \ldots, \delta_{s}\right\} \subset L \cdot M(\tilde{V}, p), \delta_{j} \rightarrow 0$ over $\bar{N}$, and $G\left(x_{1}, \ldots, x_{s}\right)$ analytic and vanishing at $x_{1}=x_{2}=\ldots=x_{s}=0$. By assembling like terms in the representation $\left(^{* *}\right)$ we can obtain either $f=0$ or $f=m_{0}\left(1+e_{0}\right)$ with $m_{0} \in L \cdot M(\tilde{V}, p)$, and $e_{0} \in \tilde{E}_{p 0}(\tilde{N}, \tilde{V})$, whence $f \in \tilde{A}_{0}(Y)$. Thus $\tilde{A}_{0}(Y) \subset$ $\widetilde{B}_{p 0}(\tilde{V}, \bar{N})$. Then since $\tilde{A}_{0}(Y)$ is obviously closed under multiplication and division, and $\widetilde{B}_{p 0}(\tilde{V}, \bar{N})$ is obviously closed under addition and subtraction, $\tilde{A}_{0}(Y)$ is a field.

\section{COLUMBia University,}

